IUCr]

ISSN 2052-2525

BIOLOGY|MEDICINE

Received 22 May 2020

Accepted 2 September 2020

Edited by $\mathrm{H}$. Chapman, DESY/Universität Hamburg, Germany

Keywords: mesophase crystallization; lipid sponge phase; monoolein; photosynthetic reaction centers; Rhodobacter sphaeroides; serial crystallography.

PDB references: Photosynthetic reaction center from Rhodobacter sphaeroides strain RV, in surfo crystallization, 6z02; LCP crystallization, $6 z 27$; LSP co-crystallization with spheroidene, $6 z 1 j$

Supporting information: this article has supporting information at www.iucrj.org

\section{Novel approaches for the lipid sponge phase crystallization of the Rhodobacter sphaeroides photosynthetic reaction center}

\author{
Georgii Selikhanov, ${ }^{\text {a,b }}$ Tatiana Fufina, ${ }^{\mathrm{b}}$ Lyudmila Vasilieva, ${ }^{\mathrm{b}}$ Christian Betzel ${ }^{\mathrm{c}, \mathrm{d}}$ and \\ Azat Gabdulkhakov ${ }^{\mathrm{a} *}$
}

\begin{abstract}
a Institute of Protein Research, Russian Academy of Sciences, Institutskaya 4, Puschino, Moscow region 142290, Russian Federation, $\mathbf{b}$ Institute of Basic Biological Problems, Russian Academy of Sciences, Institutskaya 2, Puschino, Moscow region 142290, Russian Federation, 'Institute of Biochemistry and Molecular Biology, University of Hamburg, at Deutsches Elektronen-Synchrotron (DESY), Notkestrasse 85, Hamburg, 22607, Germany, and ${ }^{\mathbf{d}}$ The Hamburg Centre for Ultrafast Imaging (CUI), Luruper Chaussee 149, Hamburg, 22761, Germany. *Correspondence e-mail: azat@vega.protres.ru
\end{abstract}

With the recent developments in the field of free-electron-laser-based serial femtosecond crystallography, the necessity to obtain a large number of highquality crystals has emerged. In this work crystallization techniques were selected, tested and optimized for the lipid mesophase crystallization of the Rhodobacter sphaeroides membrane pigment-protein complex, known as the photosynthetic reaction center (RC). Novel approaches for lipid sponge phase crystallization in comparatively large volumes using Hamilton gas-tight glass syringes and plastic pipetting tips are described. An analysis of RC crystal structures obtained by lipid mesophase crystallization revealed non-native ligands that displaced the native electron-transfer cofactors (carotenoid spheroidene and a ubiquinone molecule) from their binding pockets. These ligands were identified and were found to be lipids that are major mesophase components. The selection of distinct co-crystallization conditions with the missing cofactors facilitated the restoration of spheroidene in its binding site.

\section{Introduction}

Although water-soluble proteins can be crystallized and studied by X-ray structural analysis with relative success, membrane proteins remain problematic objects for analysis of their spatial structure. Crystallization of membrane proteins using detergents is often ineffective, since large micelles form around the proteins that prevent the formation of crystalline contacts (Bill et al., 2011). One of techniques developed for membrane protein crystallization is crystallization using lipid mesophases. In 1996, crystallization in the lipid cubic phase (LCP) was first used and the structure of bacteriorhodopsin was successfully determined (Landau \& Rosenbusch, 1996). Later on its basis lipid sponge phase (LSP) crystallization method was introduced (Wadsten et al., 2006). In both cases, matrix lipids form an environment that mimics the cell membrane, which contributes to the crystallization process. Lipid mesophase crystallization has some advantages over crystallization by the detergent-based vapor-diffusion technique. Crystals obtained by this method often have less solvent in their overall composition, higher symmetry, increased durability and, most importantly, lipid mesophase crystallization is often the only effective crystallization method for some proteins. In recent years, the proportion of new structures obtained by applying this technique has increased 
compared with detergent-based crystallization (Ishchenko, Abola et al., 2017).

Serial crystallography, especially time-resolved experiments on X-ray free-electron laser (XFEL) equipment, requires a significant number of small crystals, and crystallization in lipid mesophase has also proven to be a useful tool for sample preparation. The principle of the 'diffraction before destruction' method requires a special approach to deliver crystals to the X-ray beam, and viscous lipid carriers provide certain advantages in this regard. The LCP jet delivery system that is often used for viscous crystal carrier matrices has lower sample consumption compared with a liquid jet. To take advantage of low consumption it is possible to mix the crystal solution with a wide variety of viscous matrices with broad hydrophobic and hydrophilic compatibility (Nam, 2019). However, this additional mixing step could introduce damage and reduce the diffraction quality of the crystals, and therefore it is more convenient to grow the crystals in viscous media such as LCP or LSP at the beginning.

In this work, the photosynthetic reaction center of the purple bacteria Rhodobacter (Rba.) sphaeroides was crystallized using various crystallization techniques. This transmembrane pigment-protein complex consists of three subunits L, $\mathrm{M}$ and $\mathrm{H}$, and ten electron-transfer cofactors, including bacteriochlorophylls, bacteriopheophytins, ubiquinones, nonheme iron ions and carotenoid spheroidene (Fig. 1) (Feher et al., 1989). We used in meso crystallization in the LCP and LSP, as well as the detergent-based vapor-diffusion method (in surfo). The purpose of this work was to select the crystallization conditions and develop an efficient technique for obtaining a large number of crystals in the LSP for further use in serial crystallography. Structural data presented here were obtained via conventional rotation crystallography for rapid quality checking of the crystals obtained.

\section{Results}

2.1. Crystalliation of the photosynthetic RC and analysis of the structures obtained

Initially, for RC crystallization we used previously described methods of mesophase crystallization in the LCP (Katona et al., 2003) and in the LSP (Wadsten et al., 2006) with slightly modified conditions. For LSP crystallization we used the hanging-drop vapor-diffusion method and for LCP crystallization a microbatch crystallization approach. High-quality protein crystals for X-ray diffraction were obtained. Diffraction data from single crystals at $100 \mathrm{~K}$ were collected at the ESRF, Grenoble; spatial crystal structures with a maximum resolution of $2.1 \AA$ were resolved and refined. Furthermore, we developed new techniques for the crystallization of reaction centers in the LSP in Hamilton gas-tight glass syringes and in plastic pipetting tips to increase the number of crystals obtained (Fig. 2). These techniques are intended to be used to obtain a sufficient number of crystals for serial crystallography experiments.

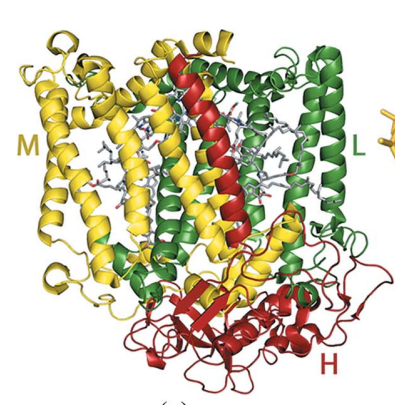

(a)

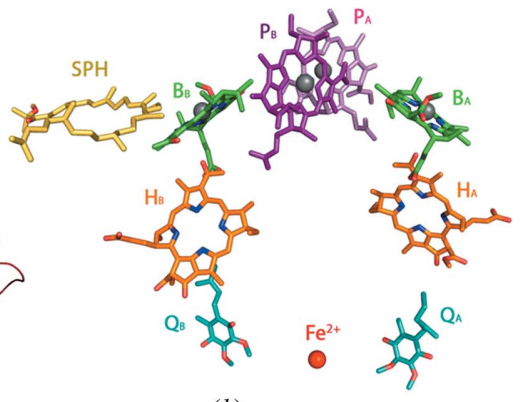

(b)
Figure 1

(a) Structure of the Rba. sphaeroides RC (PDB entry 3v3y). The L, M and $\mathrm{H}$ subunits are shown in green, yellow and red, respectively; $(b) \mathrm{RC}$ cofactors organized in two electron-transfer chains. $\mathrm{P}_{\mathrm{A}}$ and $\mathrm{P}_{\mathrm{B}}$, bacteriochlorophyll dimer; $\mathrm{B}_{\mathrm{A}}$ and $\mathrm{B}_{\mathrm{B}}$, bacteriochlorophyll monomers; $\mathrm{H}_{\mathrm{A}}$ and $\mathrm{H}_{\mathrm{B}}$, bacteriopheophytins; $\mathrm{Q}_{\mathrm{A}}$, primary ubiquinone $\mathrm{Q}_{\mathrm{B}}$, secondary ubiquinone; $\mathrm{SPH}$, spheroidene.

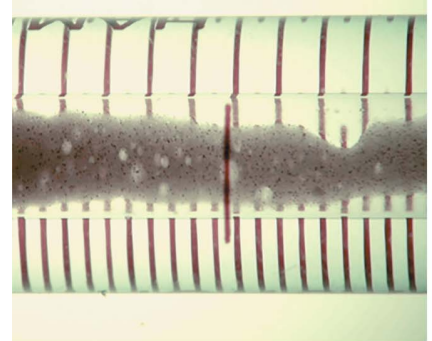

(a)

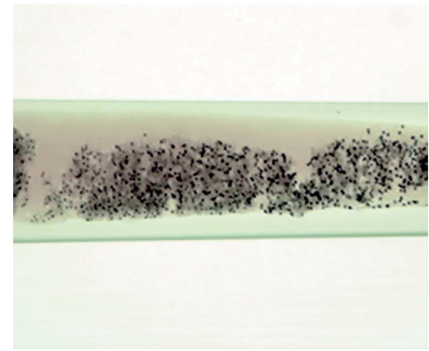

(c)

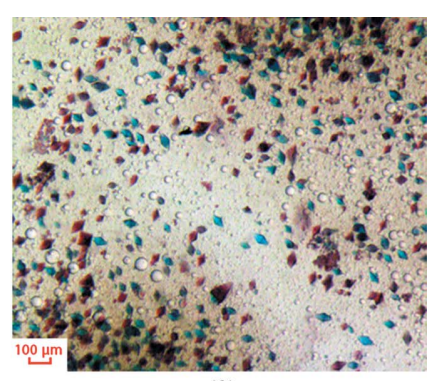

(b)

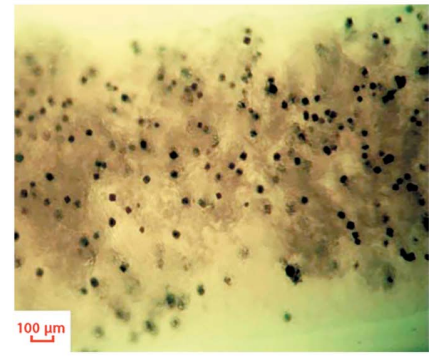

$(d)$
Figure 2

RC crystals obtained in LSP. (a) Inside a Hamilton gas-tight glass syringe; (b) a Hamilton gas-tight glass syringe under magnification; (c) inside a sealed plastic tip; $(d)$ a sealed plastic tip under magnification.

In order to have a 'control and reference' crystal structure without lipids we carried out RC crystallization by applying the detergent-based hanging-drop vapor-diffusion method utilizing conditions we used in earlier work (Gabdulkhakov et al., 2013). The resulting crystals were of sufficient quality to obtain a $2.1 \AA$ crystal structure. This in surfo structure was used for comparison with in meso structures.

It should be noted that the RC of Rba. sphaeroides is one of the most well studied membrane complexes. The number of cofactors in this RC was first determined by spectral methods during the study of the electron-transfer process and later confirmed by X-ray crystal structure analysis (Feher et al., 1989).

Analysis of spatial structures derived from RC crystals grown in the presence of lipids showed two significant differ- 
ences compared with $\mathrm{RC}$ structures obtained by in surfo vapor-diffusion crystallization.

The first difference is the absence of carotenoid spheroidene in structures derived from lipid mesophase crystals [Figs. $3(a)$ and $3(b)]$. In the carotenoid-binding region an electron density is present that resembles a carotenoid molecule. However, the size and shape of this electron density do not allow the fitting of a full-sized spheroidene molecule into it. In addition, there is a clear visible change in the protein itself. The M162 phenylalanine side group forms a kind of 'closed' conformation. In this position, the M162Phe side group occupies space that in the previously described RC structures is occupied by a spheroidene molecule.

The second difference is the presence of electron density in the secondary ubiquinone $Q_{\mathrm{B}}$ pocket, which does not correspond to a ubiquinone molecule [Figs. 3(c) and 3(d)]. In some in surfo $\mathrm{RC}$ structures this region has weak electron density, which is apparently caused by the mobility of this ubiquinone. However, there are structures in which the electron density for $Q_{\text {B }}$ is strong and clearly visible like in our 'control' crystal structure obtained without lipids. In the in meso RC structures, a different picture is observed. There is a distinct electron density in the $Q_{\mathrm{B}}$ pocket which looks unlike an ubiquinone molecule. We assume that another molecule occupies the $Q_{\mathrm{B}}$ pocket, displacing the natural ubiquinone.

\subsection{Spectral analysis of dissolved crystals}

The presence of the carotenoid molecule in the RC can be determined by the absorption spectrum in the region of this pigment-protein complex near $490 \mathrm{~nm}$. To confirm the absence of carotenoid in the RC crystals obtained by lipid mesophase crystallization, we dissolved in meso crystals and in surfo crystals in detergent-containing buffer. For that purpose we

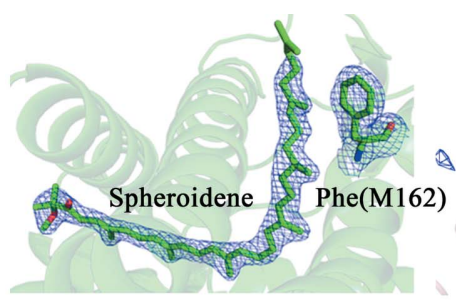

(a)

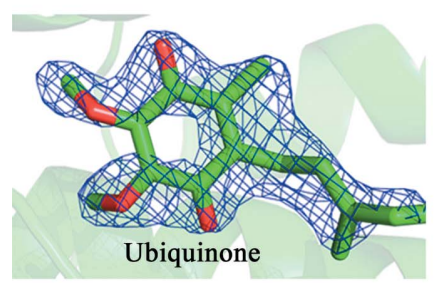

(c)

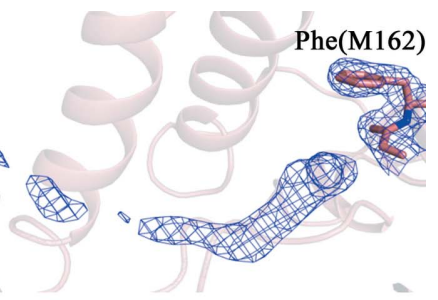

(b)

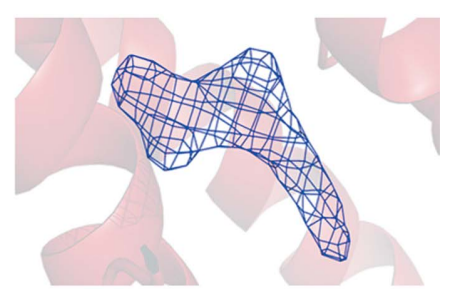

(d)
Figure 3

Fragment of the electron density map of the Rba. sphaeroides RC structure. (a) In the carotenoid binding site, under in surfo conditions, at $2.1 \AA$ resolution, $1.6 \sigma ;(b)$ in the carotenoid binding site, in the LSP, at $2.1 \AA$ resolution, $1.0 \sigma ;(c)$ in the $Q_{\mathrm{B}}$ binding site, under in surfo conditions, at $2.1 \AA$ resolution, $1.6 \sigma ;(d)$ in the $Q_{\mathrm{B}}$ binding site, in the LSP, at $2.1 \AA$ resolution, $1.0 \sigma$.

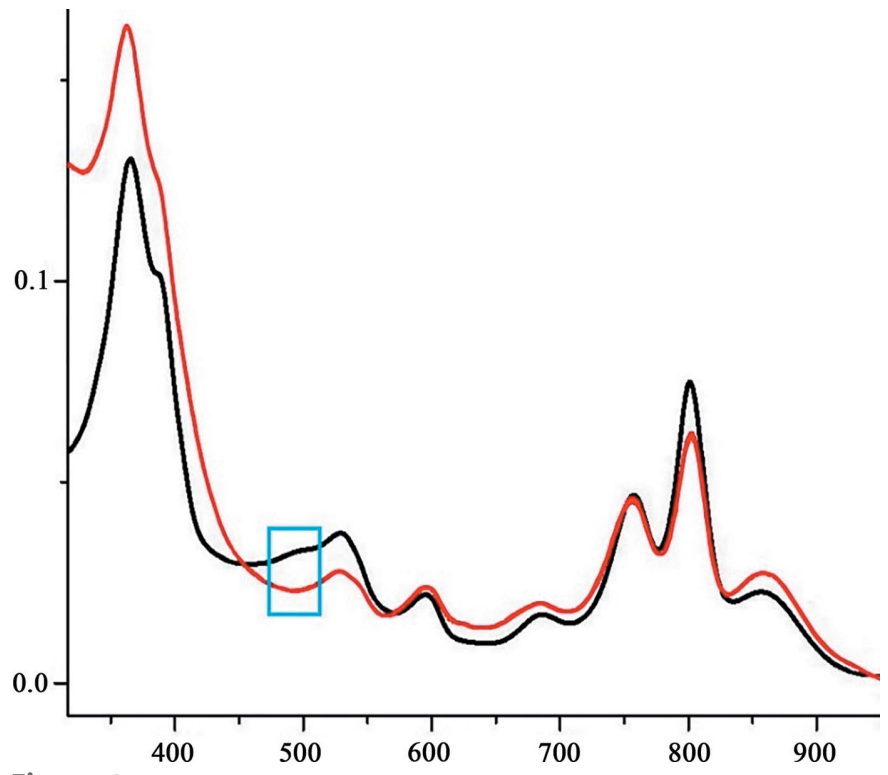

Figure 4

Absorption spectra of the Rba. sphaeroides RC solutions obtained by dissolution of in surfo crystals (black curve) and in meso crystals (red curve). The highlighted area corresponds to the carotenoid absorption region.

used crystals from the same crystallization trials from which we took samples for X-ray structural analysis. Absorption spectra of dissolved pigment-protein complexes were measured and compared (Fig. 4). It can be seen that there is a small absorption band in the region of $490 \mathrm{~nm}$ in the spectrum of the in surfo RC, characteristic of a spheroidene molecule, which is not observed in the spectrum of in meso $\mathrm{RC}$, and which is in agreement with the absence of this molecule in the in meso RCs.

\subsection{Determination of non-native ligands}

In an attempt to identify the molecules replacing the native cofactors in their binding pockets we discovered that the observed electron densities could be described well by monoolein molecules. Monoolein is the most commonly used matrix lipid for mesophase crystallization. It is a representative of monoacylglycerols (MAGs).

Apparently, owing to good steric compliance, monoolein molecules are able to penetrate the binding sites of spheroidene and secondary ubiquinone in the $\mathrm{RC}$, thus replacing the native cofactors [Figs. 5(a) and 5(b)].

\subsection{Co-crystallization with carotenoid and ubiquinone}

To solve the problem of non-native ligands, we attempted to increase the concentration of the missing cofactors in order to competitively displace the non-native ligands. It was shown that to increase the occupancy of $Q_{\mathrm{B}}$ in RC crystals, ubiquinone could be added to the mother liquor as described in the work by Axelrod et al. (2000). In these experiments the soaking method proved to be effective in the case of in surfo crystals. Another widespread crystallographic approach is a 


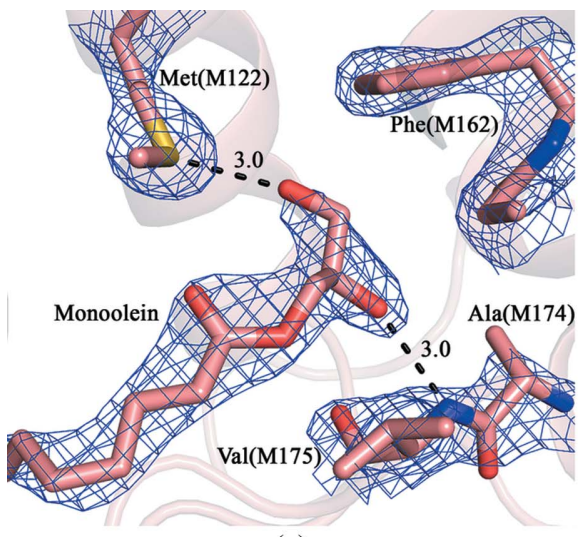

(a)

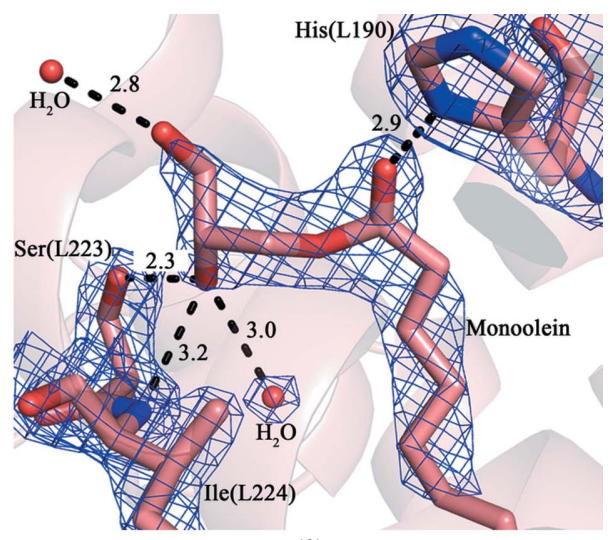

(b)
Figure 5

Monoolein molecules fitted into an electron density of interest in the Rba. sphaeroides RC $(2.1 \AA$ resolution, $1.6 \sigma)$. (a) Carotenoid binding site and $(b) Q_{\mathrm{B}}$ binding site.

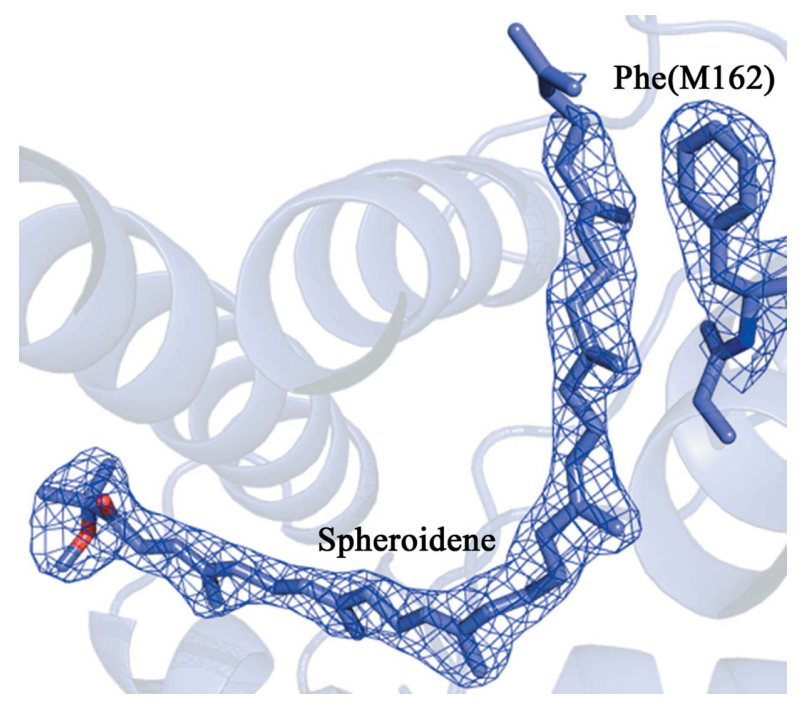

Figure 6

Fragment of the electron density map of the Rba. sphaeroides RC structure in the carotenoid binding site. The sample co-crystallized with carotenoid in the LSP $(2.1 \AA$ resolution, $1.6 \sigma)$.

co-crystallization whereby the substance is added to the crystallization mix during its preparation (Müller, 2017).

We attempted to both co-crystallize reaction centers in the LSP with the missing cofactors and soak mature in meso crystals in cofactor-containing solution. Co-crystallization and soaking were performed with the addition of spheroidene and ubiquinone solutions.

First, we tried soaking mature crystals. We added spheroidene dissolved in acetone and ubiquinone dissolved in ethyl acetate to the hanging drops that contained LSP with embedded RC crystals obtained during the vapor diffusion experiments. We tried different volumes of additives and, in the most extreme cases, we completely covered LSP with the organic solvent. We proposed that water channels that exist in LSP as well as in LCP would facilitate the delivery of cofactors to the crystals (Briggs et al., 1996; Engström et al., 1998). After three weeks of soaking, these crystals were used for diffraction data collection.
In the case of soaking with ubiquinone the samples showed no differences in their structure in the $Q_{\mathrm{B}}$ binding site compared with the control in meso structure. Therefore, the ubiquinone soaking method proved to be ineffective in the case of in meso crystallization.

For spheroidene soaking we had different results for every crystal. In some cases we saw no differences in the carotenoid binding site, and in others carotenoid completely displaced the non-native ligand. Many structures had hybrid densities that confirmed only partial displacement of the monoolein. The reason for such results may be the uneven penetration of the spheroidene solution into different parts of the LSP.

The first problem with the soaking method is that it seems to be difficult to apply on a larger scale due to uneven solvent penetration. Another problem is that most crystals are damaged by the organic solvents (both acetone and ethyl acetate) and their diffraction quality noticeably decreases.

Later co-crystallization experiments were performed. We added carotenoid solution to the crystallization mix. By titration it was shown that when the final concentration of acetone is approximately $5 \%$ or more the quality of the resulting crystals deteriorates, or they even stop appearing. At a final acetone concentration of $2.5 \%$, no negative effects on the crystallization or crystal quality were observed. By adding an acetone solution of spheroidene to the final acetone concentration of $2.5 \%$, crystals were obtained in which the spheroidene completely displaced monoolein from its binding site (Fig. 6).

A series of co-crystallization experiments were carried out with the addition of various volumes of ubiquinone solution. Even at high solvent concentration, when the crystal quality deteriorated significantly, the $Q_{\mathrm{B}}$ binding site remained occupied by monoolein. The reason for this may be the high affinity of monoolein for this binding site and/or too much monoolein in the crystallization mixture with respect to ubiquinone. Another possible reason is the relatively low affinity of ubiquinone to the $Q_{\mathrm{B}}$ binding site associated with its function: ubiquinone comes and goes easily.

2.5. Increasing the number of crystals: syringe-based and innozzle crystallization

To increase the number of crystals for further use in serial crystallography, it is necessary to enlarge the volume of crystallization mixtures.

Crystallization using lipid mesophases has a characteristic feature. The crystallization process strongly depends on the ratio of the area of the water/lipid phase interface and the volume of the crystallization mixture. For this reason, attempts 
to crystallize RC in LSP by vapor diffusion in a sitting drop failed.

In order to increase the total volume of the crystallization mixture while maintaining a sufficient phase boundary surface, reaction centers were crystallized in Hamilton gas-tight glass syringes and later in plastic tips. Both methods allowed us to obtain sample quantities suitable for serial crystallography studies. In the case of syringe crystallization the best resolution was only $2.8 \AA$. Applying the in-tips technique allowed us to obtain a resolution up to $1.8 \AA$. Syringe-based and in-tip crystallization techniques are described in detail in the Methods section.

\section{Discussion}

In this work novel crystallization techniques for lipid mesophase crystallization of the Rba. sphaeroides RC were tested and optimized, and we obtained a considerable number of high-quality crystals. This successful approach can be further used to produce samples for serial crystallography experiments. We also showed that, despite the many advantages of lipid mesophase crystallization, it has some disadvantages. We found displacements of two native cofactors by non-native ligands in the in meso structures together with changes in the spatial arrangement of some amino acid residues.

Analysis of RC structures of some purple bacteria that are currently available in the Protein Data Bank identified other cases where crystallization in the lipid mesophase led to the replacement of natural cofactors with geometrically similar molecules. The binding sites of the ubiquinone $Q_{\mathrm{B}}$ and the carotenoid spheroidene in the in meso crystal structures deposited by other authors also contain electron densities that do not correspond to the native cofactors (Fig. S1 of the supporting information). In one of these structures, obtained by crystallization in the LCP (PDB entry 1ogv; Katona et al., 2003), unidentified positive difference electron densities in the binding site of the ubiquinone $Q_{\mathrm{B}}$ [Fig. S1 $(a)$ ] and in the binding site of carotenoid (Fig. S1b) were observed. The same pattern was observed in the case of the RC structure obtained from crystallization in the LSP [Wadsten et al., 2006; PDB entry 2gnu; Figs. S1 $(c)$ and $\mathrm{S} 1(d)$ ]. The electron densities in these crystal structures are very similar to what we have observed in our structures. Figs. $\mathrm{S} 1(b)$ and $\mathrm{S} 1(d)$ show our attempt to fill the carotenoid binding site in these structures with the monoolein molecule. The electron density found in the $Q_{\mathrm{B}}$ binding site can also be attributed to the monoolein molecule [Fig. $\mathrm{S} 1(a)$ ].

In previous work, a similar feature of the mesophase structure of the RC from purple bacterium Blastochloris (Bl.) viridis, which was crystallized in LSP, was observed (Wöhri et al., 2009). This photosynthetic RC is similar to the RC of Rba. sphaeroides, except for the presence of the additional subunit C. The resulting structure revealed the presence of a nonnative cofactor in the binding region of the secondary ubiquinone $Q_{\mathrm{B}}$. The authors described this feature and fit in a molecule they consider to be monoolein but when viewed from the crystal structure is actually an MPG $\{[(\mathrm{Z})$-octadec-9- enyl] (2R)-2,3-bis(oxidanyl)propanoate compound which is probably the result of a mistake in the HIC-Up database where the MPG molecule was described as a monoolein molecule (PDB entry 2wjm, Fig. S2). In this RC a 15-cis-1,2dihydroneurosporene molecule is normally located in the carotenoid binding pocket. It is present in both in meso and in surfo crystal structures (Wöhri et al., 2009; Li et al., 2006). Thus, in the lipid mesophase crystal structure of the $B l$. viridis photosynthetic RC no anomalies in the carotenoid binding region have been observed.

The Rba. sphaeroides RC is a prospective object for structural and functional studies of photosynthesis. Since the object is photoactive, it is highly suitable for time-resolved serial investigations of the photochemical charge separation with no need for additional modifications. However, since the spheroidene and secondary ubiquinone are important for the proper functioning of this pigment-protein complex, their absence may cause problems for studies of photosynthetic processes in crystals obtained by crystallization in lipid mesophases.

One way to solve the problem of substitution or loss of natural cofactors may be an increase in their concentration in the crystallization mixture, which may help to displace the alien components from protein binding sites. The possibility of this approach was demonstrated here using RC co-crystallization with spheroidene. However, we could not succeed in restoring ubiquinone in the $Q_{\mathrm{B}}$ site probably due to its relatively low affinity to this site (which is closely related to its function) in comparison with monoolein. Another possible way is to replace monoolein with another suitable matrix lipid that has a different structure. To date, a number of studies have already shown the fundamental potential of such approach (Li et al., 2011; Höfer et al., 2011; Salvati Manni et al., 2015; Ishchenko, Peng et al., 2017; Borshchevskiy et al., 2010).

Rba. sphaeroides RC crystals without secondary ubiquinone are still of interest to study the ultrafast (from femotoseconds to picoseconds) steps of electron transfer from the bacteriochlorophyll dimer to the primary ubiquinone by time-resolved serial crystallography. The absence of ubiquinone $Q_{\mathrm{B}}$ makes it difficult to study only the last stage of the primary electron transfer from $Q_{\mathrm{A}}$ to $Q_{\mathrm{B}}$. Crystals obtained in this work were successfully tested using silicon microchips for fixed-target sample delivery, both at synchrotron beamlines and at the EuXFEL SFX station, and the results will be described and published elsewhere.

\section{Materials and methods}

\subsection{Protein preparation and purification}

The recombinant strain of $R b a$. sphaeroides that produced wild-type reaction centers was cultivated using Huttner medium containing kanamycin $\left(5 \mu \mathrm{g} \mathrm{ml}^{-1}\right)$ and tetracyclin $\left(1 \mu \mathrm{g} \mathrm{ml}^{-1}\right)$ as described previously (Khatypov et al., 2005). Reaction centers were isolated and purified using two-step ion-exchange chromatography as described in the work by Fufina et al. (2007). 
After purification the RCs were solubilized in $20 \mathrm{~m} M$ Tris$\mathrm{HCl}$ buffer (pH 8.0) containing $0.1 \% N, N$-dimethyldodecylamine- $N$-oxide (LDAO). Room-temperature absorption spectra of the RC were recorded with a Shimadzu UV1800 PC spectrophotometer (Japan). Sample purity $A_{280} / A_{800}$ was $<1.4$.

\subsection{Crystallization}

For the crystallization we used 1-mono-oleyl-rac-glycerol (MO) of $99 \%$ purity and the 1,2,3-heptanetriol high melting point isomer ( $>98 \%$ purity) purchased from Sigma-Aldrich; Jeffamine M600 was purchased from Hampton Research and LDAO ( $>99 \%$ purity) was purchased from Sigma-Aldrich.

For crystallization experiments we used RC solutions with a protein concentration of $25-30 \mathrm{mg} \mathrm{ml}^{-1}$. The lipidic sponge phase was prepared by mixing lipidic cubic phase and buffer solution, as described in the work by Wadsten et al. (2006).

For RC crystallization, we used the previously described mesophase crystallization in the LCP (Katona et al., 2003) and in the LSP (Wadsten et al., 2006). For LSP crystallization trials we used the hanging-drop vapor-diffusion method and for the LCP trials we used microbatch crystallization. Diffraction data were collected at the ESRF in Grenoble and at PETRA III (DESY) in Hamburg. In addition, we carried out RC crystallization by vapor diffusion in a hanging drop with the addition of detergent under the conditions established in our earlier work (Gabdulkhakov et al., 2013).

\subsection{Dissolving crystals for spectroscopic analysis}

The crystals were dissolved in $200 \mu \mathrm{l} 20 \mathrm{~m} M$ Tris- $\mathrm{HCl}$ buffer $\mathrm{pH} 8.0$ containing 1\% LDAO. Samples were then centrifuged for $2 \mathrm{~min}$ at $5000 \mathrm{~g}$ to remove undissolved RC crystals. Optical absorption spectra of the isolated RCS were measured using a Shimadzu UV-1800 PC spectrophotometer (Japan): the path length was $10 \mathrm{~mm}$. Sodium ascorbate was added at $1 \mathrm{~m} M$ final concentration in order to keep the primary donor in the reduced state.

\subsection{Crystallization in Hamilton glass syringes}

For this technique we used 100 and $250 \mu \mathrm{l}$ Hamilton gastight glass syringes. At first we mixed LSP with a protein solution (1:1 ratio to a final volume of about $80 \mu \mathrm{l}$ ) inside two connected $100 \mu$ syringes until the mixture was homogeneous. Finally LSP/protein mix was transferred into one syringe. Another syringe was disconnected from the connector. A $250 \mu \mathrm{l}$ syringe was filled with a salt counter solution (2M trisodium citrate). After disposing of the residual air in the syringe with salt solution, it was connected to the LSPcontaining syringe. The LSP/protein mix was smoothly injected into the salt solution.

The syringe containing salt solution and the LSP/protein mixture was positioned horizontally allowing LSP to be spread in a thin layer. Incubation was carried out in a syringe wound with Parafilm in the dark at $16^{\circ} \mathrm{C}$. RC crystals began to form on the second day, and reached their maximum size after $7 \mathrm{~d}$. In this way, we were able to obtain a homogeneous mixture of crystals [Figs. 2(a) and 2(b)]. However, this approach only allowed us to obtain crystals that could diffract to a maximum resolution of $2.8 \AA$.

\subsection{In-tip crystallization}

To increase the quality of the crystals, we established a new approach of mesophase crystallization. Instead of glass syringes, we used 1-10 $\mu$ l plastic tips for automatic pipettes to incubate the crystallization mixture (Fig. S3). Positioned horizontally, they allow a vast area of the phase boundary to be saved, while, due to the cheapness and availability of the tips, we optimize crystallization conditions faster and more efficiently than upon crystallization in syringes. The scheme of this experiment is presented in Fig. S4. In-tip crystallization allowed titration of the salt solution concentration and control of its volume ratio to the LSP. Injection of salt solution and LSP/protein mix inside the tip was performed using Hamilton gas-tight syringes. To isolate the crystallization mixture from ambient air, the narrow end of the tip was first sealed, and after adding all the components of the mixture, the wide end of the tip was tightly wrapped in Parafilm.

We used trisodium citrate with concentrations from 1 to $2 M$ as a salt counter solution. We selected crystallization conditions in which large crystals (about $70 \mu \mathrm{m}$ ) suitable for X-ray diffraction experiments were formed. Diffraction data were collected and the crystal structure was refined up to $2.1 \AA$. By applying different conditions larger quantities of smaller crystals (about $20 \mu \mathrm{m}$ ) were obtained [Figs. 2(c) and 2(d)]. These crystals were tested and they diffracted up to $1.8 \AA$ resolution. Such crystals can be used for serial diffraction data collection. The maximum volume of LSP containing crystals we obtained from one tip was about $30 \mu$, which is less than the maximum volume from Hamilton syringes, but much larger than when using the hanging-drop method (about $1 \mu \mathrm{l}$ of crystal-filled LSP per drop). To extract crystals from the tip, the tip was cut off and either the LSP was removed with another tip, or the tip was put on an automatic pipette and the mixture was squeezed out.

\subsection{Preparation of spheroidene and ubiquinone}

Both spheroidene and ubiquinone were dissolved in suitable organic solvents. To dissolve spheroidene we used acetone. The extraction method for this pigment is described in the work by Ashikhmin et al. (2017).

Ethyl acetate was chosen to dissolve ubiquinone, as it is one of the most effective solvents for this substance (Zhao et al., 2013). We dissolved ubiquinone (Coenzyme Q10) powder (Sigma-Aldrich) in ethyl acetate until the solution was completely saturated.

\subsection{X-ray diffraction analysis}

X-ray diffraction data were collected at the BL14.1 beamline at BESSY II (Berlin, Germany) at $100 \mathrm{~K}$ using a crystal grown under in surfo conditions.

Diffraction data from the LCP were collected on the ID23-1 beamline at the European Synchrotron Radiation Facility (ESRF), Grenoble, France (Nurizzo et al., 2006), equipped 
Table 1

Data collection and processing.

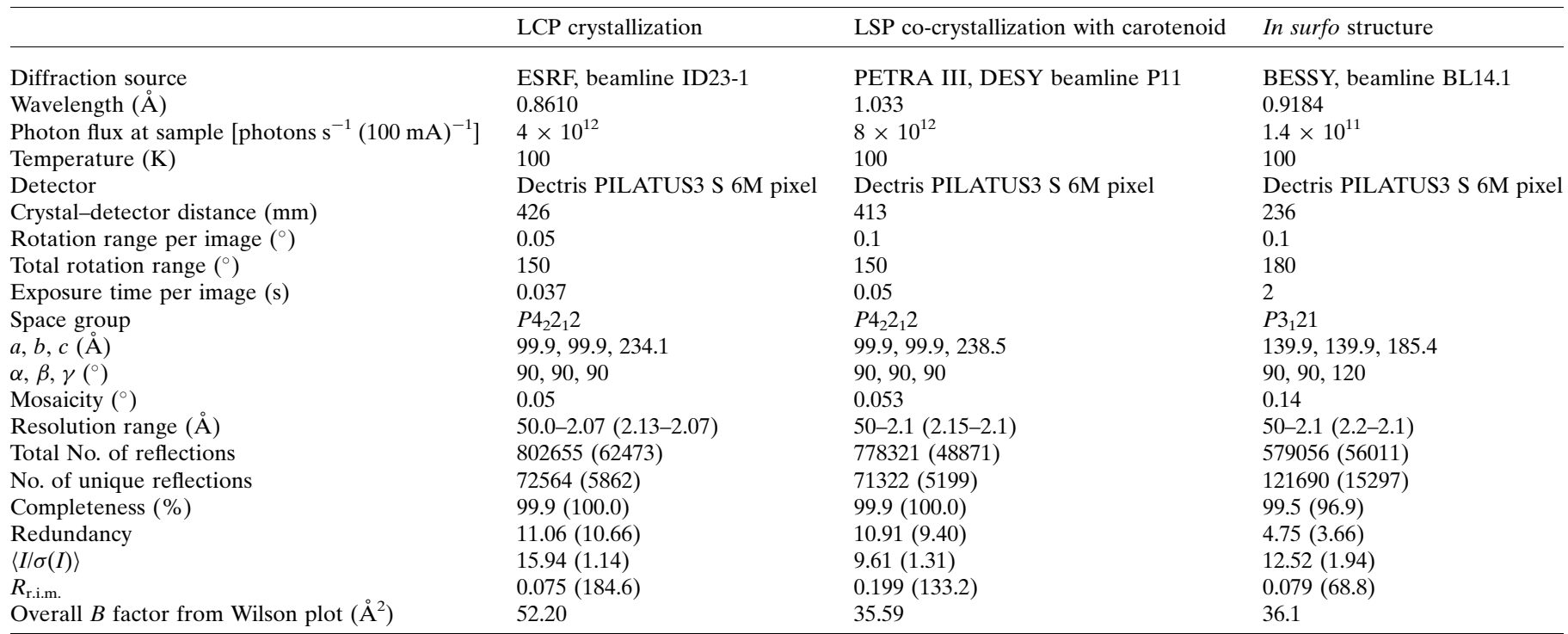

Table 2

Structure solution and refinement.

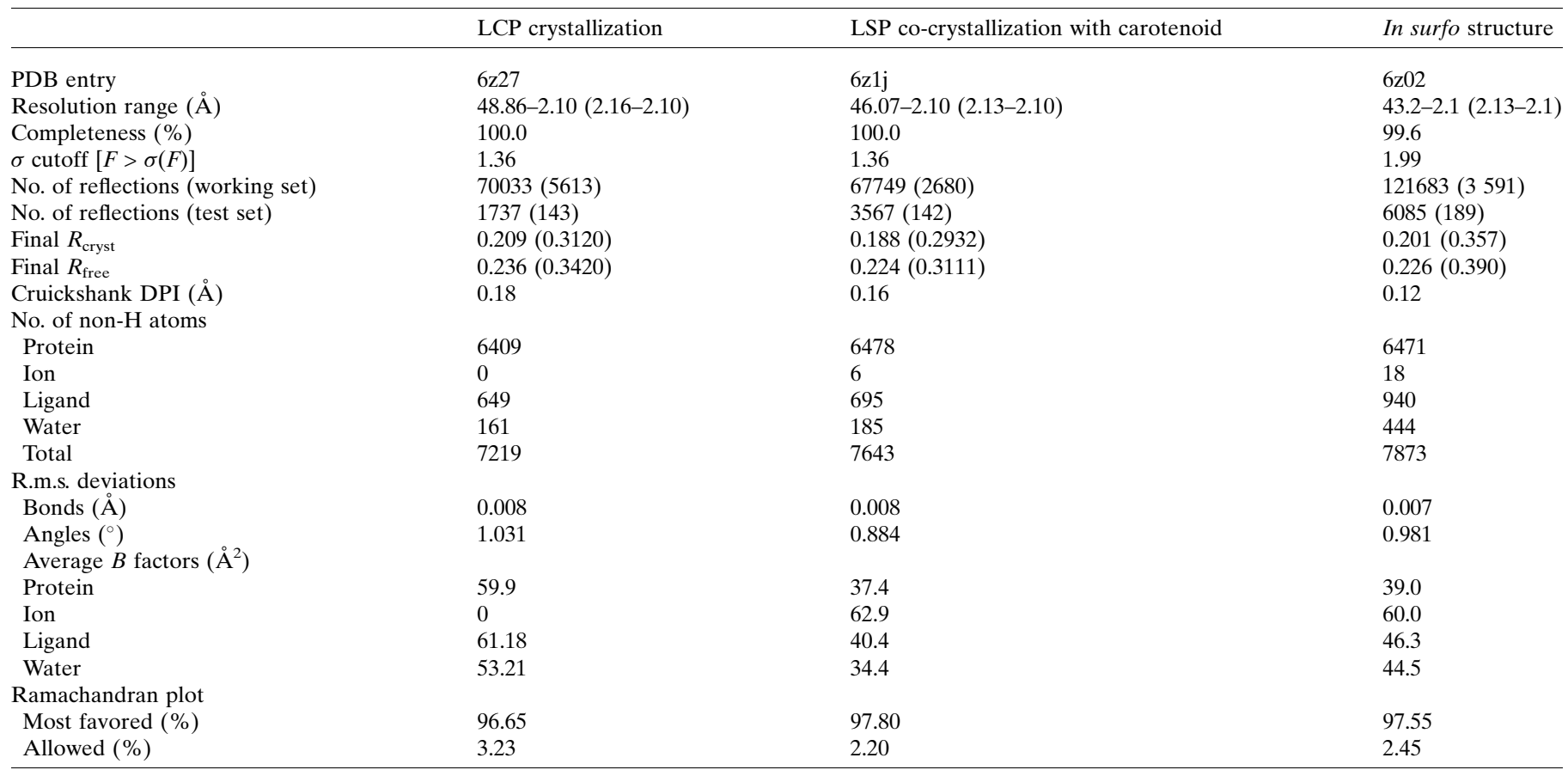

with a PILATUS 6M detector. Data collection was controlled by the $M x C u B E$ system (Gabadinho et al., 2010) and the strategy was calculated by BEST (Bourenkov \& Popov, 2010).

A dataset from the RC crystallized in LSP with carotenoid was collected at beamline P11 at PETRA III (DESY, Hamburg, Germany). All data were processed and scaled with the $X D S$ package (Kabsch, 2010).

The structures were solved by molecular replacement using Phaser (McCoy et al., 2007) and the structure of the photo- synthetic RC from Rba. sphaeroides strain RV (PDB entry 3v3y; Vasilieva et al., 2012) was used as a search model. The initial model was subjected to crystallographic refinement using REFMAC5 (Murshudov et al., 2011). Manual rebuilding of the model was carried out using Coot (Emsley et al., 2010). Data and refinement statistics are summarized in Tables 1 and 2. The coordinates and structure factors have been deposited in the Protein Data Bank with the PDB entries 6z02 and 6z1j. Figures were prepared using PyMOL (DeLano, 2002). 


\section{Acknowledgements}

We express our gratitude to Aleksandr Ashikhmin for providing us with the spheroidene solution.

\section{Funding information}

This study was supported by the program of the Presidium of the Russian Academy of Sciences 'Molecular and Cell Biology and Postgenomic Technologies' and the Russian Foundation for Basic Research (grant Nos. 18-02-40008 and 17-00-00207). $\mathrm{CB}$ acknowledges financial support by the Cluster of Excellence 'Advanced Imaging of Matter' of the Deutsche Forschungsgemeinschaft (DFG) - EXC 2056 - (project No. 390715994) and support by BMBF (project Nos. 50WB1422 and 05K16GUA); Deutsche Forschungsgemeinschaft (award No. 390715994 to CB); Bundesministerium für Bildung und Forschung (award Nos. 50WB1422 and 05K16GUA).

\section{References}

Ashikhmin, A., Makhneva, Z., Bolshakov, M. \& Moskalenko, A. (2017). J. Photochem. Photobiol. B, 170, 99-107.

Axelrod, H. L., Abresch, E. C., Paddock, M. L., Okamura, M. Y. \& Feher, G. (2000). Proc. Natl Acad. Sci. USA, 97, 1542-1547.

Bill, R. M., Henderson, P. J. F., Iwata, S., Kunji, E. R. S., Michel, H., Neutze, R., Newstead, S., Poolman, B., Tate, C. G. \& Vogel, H. (2011). Nat. Biotechnol. 29, 335-340.

Borshchevskiy, V., Moiseeva, E., Kuklin, A., Büldt, G., Hato, M. \& Gordeliy, V. (2010). J. Cryst. Growth, 312, 3326-3330.

Bourenkov, G. P. \& Popov, A. N. (2010). Acta Cryst. D66, 409-419.

Briggs, J., Chung, H. \& Caffrey, M. (1996). J. Phys. II Fr. 6, 723-751.

DeLano, W. L. (2002). PyMOL. http://www.pymol.org.

Emsley, P., Lohkamp, B., Scott, W. G. \& Cowtan, K. (2010). Acta Cryst. D66, 486-501.

Engström, S., Alfons, K., Rasmusson, M. \& Ljusberg-Wahren, H. (1998). The Colloid Science of Lipids, Vol. 108, edited by B. Lindman \& B. W. Ninham, pp. 93-98. Darmstadt: Steinkopff.

Feher, G., Allen, J. P., Okamura, M. Y. \& Rees, D. C. (1989). Nature, 339, 111-116.

Fufina, T. Y., Vasilieva, L. G., Khatypov, R. A., Shkuropatov, A. Ya. \& Shuvalov, V. A. (2007). FEBS Lett. 581, 5769-5773.

Gabadinho, J., Beteva, A., Guijarro, M., Rey-Bakaikoa, V., Spruce, D., Bowler, M. W., Brockhauser, S., Flot, D., Gordon, E. J., Hall, D. R., Lavault, B., McCarthy, A. A., McCarthy, J., Mitchell, E., Monaco, S., Mueller-Dieckmann, C., Nurizzo, D., Ravelli, R. B. G.,
Thibault, X., Walsh, M. A., Leonard, G. A. \& McSweeney, S. M. (2010). J. Synchrotron Rad. 17, 700-707.

Gabdulkhakov, A. G., Fufina, T. Y., Vasilieva, L. G., Mueller, U. \& Shuvalov, V. A. (2013). Acta Cryst. F69, 506-509.

Höfer, N., Aragão, D., Lyons, J. A. \& Caffrey, M. (2011). Cryst. Growth Des. 11, 1182-1192.

Ishchenko, A., Abola, E. E. \& Cherezov, V. (2017). Protein Crystallography, Vol. 1607, edited by A. Wlodawer, Z. Dauter \& M. Jaskolski, pp. 117-141. New York: Springer.

Ishchenko, A., Peng, L., Zinovev, E., Vlasov, A., Lee, S. C., Kuklin, A., Mishin, A., Borshchevskiy, V., Zhang, Q. \& Cherezov, V. (2017). Cryst. Growth Des. 17, 3502-3511.

Kabsch, W. (2010). Acta Cryst. D66, 125-132.

Katona, G., Andréasson, U., Landau, E. M., Andréasson, L.-E. \& Neutze, R. (2003). J. Mol. Biol. 331, 681-692.

Khatypov, R. A., Vasilieva, L. G., Fufina, T. Y., Bolgarina, T. I. \& Shuvalov, V. A. (2005). Biochemistry, 70, 1256-1261.

Landau, E. M. \& Rosenbusch, J. P. (1996). Proc. Natl Acad. Sci. USA, 93, 14532-14535.

Li, D., Lee, J. \& Caffrey, M. (2011). Cryst. Growth Des. 11, 530-537.

Li, L., Mustafi, D., Fu, Q., Tereshko, V., Chen, D. L., Tice, J. D. \& Ismagilov, R. F. (2006). Proc. Natl Acad. Sci. USA, 103, 1924319248.

McCoy, A. J., Grosse-Kunstleve, R. W., Adams, P. D., Winn, M. D., Storoni, L. C. \& Read, R. J. (2007). J. Appl. Cryst. 40, 658-674.

Müller, I. (2017). Acta Cryst. D73, 79-92.

Murshudov, G. N., Skubák, P., Lebedev, A. A., Pannu, N. S., Steiner, R. A., Nicholls, R. A., Winn, M. D., Long, F. \& Vagin, A. A. (2011). Acta Cryst. D67, 355-367.

Nam, K. (2019). Int. J. Mol. Sci. 20, 1094.

Nurizzo, D., Mairs, T., Guijarro, M., Rey, V., Meyer, J., Fajardo, P., Chavanne, J., Biasci, J.-C., McSweeney, S. \& Mitchell, E. (2006). J. Synchrotron Rad. 13, 227-238.

Salvati Manni, L., Zabara, A., Osornio, Y. M., Schöppe, J., Batyuk, A., Plückthun, A., Siegel, J. S., Mezzenga, R. \& Landau, E. M. (2015). Angew. Chem. Int. Ed. 54, 1027-1031.

Vasilieva, L. G., Fufina, T. Y., Gabdulkhakov, A. G., Leonova, M. M., Khatypov, R. A. \& Shuvalov, V. A. (2012). Biochim. Biophys. Acta, 1817, 1407-1417.

Wadsten, P., Wöhri, A. B., Snijder, A., Katona, G., Gardiner, A. T., Cogdell, R. J., Neutze, R. \& Engström, S. (2006). J. Mol. Biol. 364, 44-53.

Wöhri, A. B., Wahlgren, W. Y., Malmerberg, E., Johansson, L. C., Neutze, R. \& Katona, G. (2009). Biochemistry, 48, 9831-9838.

Zhao, Y., Sun, Y.-H., Li, Z.-Y., Xie, C., Bao, Y., Chen, Z.-J., Gong, J.B., Yin, Q.-X., Chen, W. \& Zhang, C. (2013). J. Solution Chem. 42, 764-771. 